\title{
Responsabilidad social y empresa sostenible
}

\author{
Anna Bajo Sanjuán \\ Universida Pontificia de Comillas \\ Marta González Álvarez \\ Universida Pontificia de Comillas \\ José Luís Fernández Fernández \\ Universida Pontificia de Comillas
}

\section{Palabras clave}

Responsabilidad Social, empresa, sostenibilidad, empresa sostenible, RSC, RSE

\section{Resumen}

Las organizaciones empresariales, incluidas las de medios, cumplen un papel determinante en la sociedad, especialmente en el ámbito de la globalización donde se multiplican los efectos de manera exponencial en múltiples dimensiones no sólo económicas, sino también sociales y medioambientales. La actuación de toda empresa, independientemente de su tamaño, sector o mercado provoca una serie de impactos, positivos y negativos, de los que irremediablemente ha de responder ante una sociedad cada vez más exigente. Esta responsabilidad debe ser asumida desde una conciencia del ejercicio ético que, lo quiera o no, la empresa está obligada a llevar a cabo para legitimar su existencia. El artículo que aquí se presenta ha de ayudar al lector a conocer más a fondo qué es eso llamado Responsabilidad Social de la Empresa, a qué agentes de la sociedad afecta, cuáles son las motivaciones que empujan a las organizaciones a incorporarla a su gestión, de manera estratégica, así como los mecanismos e iniciativas de impulso que propician su implantación. Concluimos con una reflexión sobre el pobre balance que la incorporación de la RSE está teniendo en las empresas, en general, salvo una representativa minoría que parece haber entendido que, sobre todo en estos tiempos de crisis, no cabe otro camino para la subsistencia y el éxito en el largo plazo que la apuesta por la sostenibilidad. 


\title{
Corporate Responsibility and Sustainable Enterprise
}

\section{Keywords}

Social Responsibility, Corporate Social Resposibility, company, sustainability, sustainable enterprise, CSR

\begin{abstract}
Business organizations, including those in the media sector, fulfill an important role in society, particularly within the scope of globalization where their impacts multiply exponentially in several dimensions such as the economic, social and environmental ones. The actions of every business, irrespective of size, sector or market, generate both positive and negative impacts, for which they are certain to become accountable to an increasingly demanding society. This responsibility must be taken from the conscience of ethical practice, which whether they like it or not, businesses are forced to conduct in order to legitimise their existence. The article which we are presenting should provide the reader a deeper knowledge of the concept of Corporate Social Responsibility, the social actors that are affected by it and the motivations that drive organisations to incorporate it as a strategic part of their management as well as the initiatives and mechanisms that favor its establishment. We finalise with a reflection on the so far generally poor result of incorporating CSR into the corporate world except for a representative minority which seems to have understood that, particularly in times of crisis, betting on sustainability is the only path to subsistance and long term success.
\end{abstract}

\section{Autor}

Anna Bajo Sanjuán es Licenciada en Ciencias Económicas y Empresariales y Executive MBA por el Instituto de Empresa. Imparte clases en la Universidad Pontificia de Comillas e investiga en temas relacionados con la RSE y la Sostenibilidad sobre los que está desarrollando su tesis doctoral.

Marta González Álvarez es Licenciada en Psicología y cursa actualmente su doctorado en la Universidad Pontificia Comillas, en el campo de la RSE y la Ética Empresarial. Posee una amplia experiencia en consultaría siendo socia fundadora de RSE-Alcance.

José Luis Fernández Fernández es Doctor en Filosofía y profesor ordinario de la Universidad Pontificia Comillas (ICADE). Dirige la Cátedra de Ética Económica y Empresarial y es miembro destacado de diferentes iniciativas de promoción e investigación de la RSE y la Ética Empresarial, tales como EBEN-España, Forética, Caux Round Table. Además es miembro del Consejo Editorial de distintas publicaciones en la materia. 


\section{Un modelo de empresa sostenible para un escenario complejo}

No pretendemos describir por menudo el crítico panorama en el que vivimos. Simplemente, querríamos empezar este trabajo de marcado tono conceptual, tomando noticia de algunos de los rasgos más evidentes del contexto en que emerge la preocupación por la Responsabilidad Social de la Empresa (RSE) y la sostenibilidad, no sólo de la empresa, sino del sistema económico en su conjunto. Tras ello, estaremos en condiciones de precisar de manera afinada el tenor, el devenir del proceso los logros y los retos de la RSE. A grandes rasgos, cabe dejar sentado lo siguiente.

Vivimos en un mundo multicultural y globalizado, donde las empresas operan a escala planetaria, en mercados cada vez menos sujetos a constricciones legales, donde el peso del sector público va disminuyendo al tiempo que aumenta el protagonismo de los mercados de capitales. Esta liberalización conduce a una mayor competencia, y ésta conlleva una mayor velocidad de rotación, tanto de negocios, como de productos, técnicas comerciales, etc... para tratar de salvar la homogeneización de la oferta. Y esto es así, porque las empresas son conscientes de que, en las decisiones de compra, no sólo se valoran los activos tangibles del producto, sino también los intangibles.

Para dar respuesta a estos nuevos mercados vienen apareciendo nuevos modelos organizativos, donde, entre otras cosas, se buscan estructuras más flexibles de adaptación al mercado y mecanismos eficientes para captar y retener talento, clave para el éxito empresarial y ventaja competitiva y diferencial.

Desde otro ángulo, hay que anotar el hecho de que la sociedad ha ido tomando conciencia de la limitación de los recursos naturales y desde esta sensibilidad los consumidores están elevando el listón de las exigencias a las empresas que no actúan acorde a un comportamiento responsable, sometiéndolas incluso a boicots o presentando denuncias judiciales contra ellas.

Por otra parte, merced al poder que han ganado tanto algunos colectivos sociales (ONG, Asociaciones, Sindicatos, Fundaciones, etc.) como los medios de comunicación, sus exigencias de transparencia son cada más escuchadas por las empresas, muy vulnerables a los controles férreos y las denuncias que estos agentes que configuran el Tercer Sector vienen realizando con creciente interés y profesionalidad. Algunos escándalos financieros destapados en la última década han abierto los ojos a todo un mercado que ya no parece dispuesto a perdonar la opacidad, ni tan siquiera la sombra de la duda.

Éste es el contexto de la RSE, fenómeno relativamente reciente entre nosotros; y cuyo impacto en las organizaciones está dejándose sentir de manera inequívoca. No olvidemos que el año 1999 marcó un hito, al constituir el pistoletazo de salida de mucho de lo que a lo largo de la siguiente decena larga de años nos hubo de ocupar en el discurso de la Responsabilidad Social de la Empresa. El balance que cabe hacer, atentos a un análisis desapasionado de la realidad y de 
los datos empíricos y contrastables con los que a día de hoy contamos, por lo que a la RSE respecta, es que, sin duda, estamos en presencia de un fenómeno parcial; paradójico a todas luces; decididamente insuficiente. Sin embargo, lo conseguido hasta el día de hoy no es, en modo alguno, despreciable. Hay intuiciones, algunas buenas prácticas y ciertos principios y criterios de actuación muy aprovechables. Ello sea dicho, sin perjuicio de tener que afirmar que es necesario, en todo caso, avanzar, superar lo alcanzado y cubrir las lagunas que observamos en el ámbito de la RSE.

En un trabajo anterior (Andreu y Fernández, 2011), se indicaba una posible vía para hacer evolucionar el concepto y la realidad de la RSE hacia una creación de valor corporativo sostenible. Remitimos allí al lector interesado para que pondere la validez de la intuición allí explicitada: la RSE debiera evolucionar conceptual y prácticamente hacia un modelo de empres ayude negocio a la altura del presente. Y, a nuestro entender, lo que tras el concepto de sostenibilidad anida, entendido en un sentido amplio, puede dar sugerentes pistas para avanzar.

Pues, estamos convencidos de que la empresa es mucho más de lo que el modelo implícito en buena parte de la praxis de la gestión al uso da por asumido $\mathrm{y}$, en todo caso, algo distinto a lo que el paradigma vigente en la ciencia normal entiende por tal. La empresa es, junto a -y además de todo- lo que, con más o menos acierto, se viene a subrayar desde la ciencia normal en vigor, una institución socioeconómica y política que, a su manera, contribuye y está llamada a contribuir aún más decididamente en el futuro - naturalmente, junto a otras instancias y ámbitos de la vida social- a la configuración de un escenario desde el que se avance hacia cotas crecientes de desarrollo humano. Y ello, sin dejar de ser empresa; sino más bien, tratando de serlo más y mejor, en virtud de una suerte de proceso de toma de conciencia respecto a cuáles hayan de ser identificados como rasgos esenciales del fenómeno empresarial, convertidos, así, en auténtico principio y fundamento, en verdadero núcleo de la misión sobre el que profundizar y seguir progresando en el futuro en pos de la excelencia.

A lo largo del siglo XX se han ido proponiendo distintas teorías con la pretensión de explicar la realidad y el comportamiento de la empresa. Pues, en efecto, detrás de cada definición de empresa, hay una teoría; y, por supuesto, también detrás de cada una de ellas, de manera más o menos explícita, se asume de modo cuasi normativo - esto es, como más o menos adecuado y deseable - un modelo determinado de gestión empresarial y de la acción humana en el contexto organizativo

Dependiendo de qué metáforas se utilicen para referirse a la empresa - la jungla, la máquina de hacer dinero, el juego, el campo de batalla...- (Solomon, 1999), de qué imagen pre reflexiva se asuma respecto a lo que una empresa sea - entre otras, y sin ánimo de exhaustividad: imagen mecánico-ingenieril de la organización en línea con Taylor (1911); imagen psicologista (Murray, 1938); imagen sociologista (McGregor, 1960); imagen política (Weber, 1968)— así se 
estará en mejores o peores condiciones de articular una visión antropológica holística y completa que, sin dejar fuera de consideración ninguno de los elementos clave de la persona, contribuya a un ejercicio de la gestión más en línea con lo que la consideración ética del proceso demandaría.

Entre las teorías de empresa más comúnmente invocadas, procede citar las siguientes: Teoría de los Costes de Transacción, Teoría Neoclásica o de la Firma, Teoría Conductista, Teoría Contractual o de la Agencia, Teoría de la Empresa como Sistema. Cada una de estas aproximaciones, ilumina parcelas de interés respecto al fenómeno empresarial, pero dejan al margen de sus consideraciones otros aspectos relevantes. Estas deficiencias y lagunas harán necesario y deseable, en su momento, la elaboración de un marco conceptual más comprensivo; $y$, por esa vía, estaremos abriendo la puerta, de manera tentativa a un nuevo paradigma de empresa y de gestión, más adecuado y más en sintonía con la altura de los tiempos.

Desde hace unas décadas, es perceptible por parte de buena parte de los teóricos de management y de bastantes académicos un deslizamiento el denominado enfoque stakeholder o de base ampliada de la empresa. Se da por asumido que un planteamiento tal tiene la virtualidad respecto a las otras teorías de expresar y de poder conceptualizar de manera mucho más real y precisa la compleja red de interacciones existentes entre las empresas y las organizaciones, por una parte, y el resto de instituciones que configuran el entorno sociopolítico y cultural, por otra.

Aunque el concepto de stakeholder, como neologismo que apunta a una metáfora -literalmente vendría a significar: «el que mantiene una apuesta»... y nótese bien su cercanía fónica al término stockholder, que vale por shareholder y que sería, vertido al castellano, «el que tiene en su poder una parte alícuota de una empresa»; o sea: el accionista-, había sido utilizado ya en los años sesenta y setenta, es Edward Freeman (1984) quien sistematiza y delinea una manera de aproximarse a los stakeholders que puede dar, como resultado final, toda una teoría de la empresa y la gestión, abriendo pistas — tímidamente, si se quiere; pero abriendo pistas al fin y al cabo- hacia lo que venimos denominando un nuevo paradigma de empresa a la altura de los requerimientos y circunstancias del siglo XXI. Y si no queremos ser tan osados en la adjetivación y aplicamos un poco de sordina a la tesis, cuando menos lo que es innegable es que la teoría del stakeholder constituye una feliz metáfora que puede ponernos en pista para avanzar hacia una situación en que las empresas y el propio sistema capitalista contribuyan a configurar una realidad más justa y beneficiosa para todos. Mimbres para ello parece haberlos: es cuestión de sustituir asunciones tradicionalmente llevadas a efecto desde el planteamiento neoclásico respecto al sistema capitalista por otras alternativas.

Se entiende por stakeholder cualquier individuo o grupo de interés que, de alguna manera puede afectar o ser afectado por la consecución de los objetivos 
empresariales (Freeman, 1984: 24). Por consiguiente, la dirección estratégica de la empresa debiera atender no sólo a la consecución de los objetivos de los dueños y accionistas, sino de un más amplio abanico de interesados: trabajadores, clientes, sociedad en su conjunto, proveedores, etc. Viendo a ser esto una especie de condición de posibilidad de la supervivencia de la empresa a largo plazo, toda vez que, como hemos dicho en otro lugar, el objetivo de la gestión y la razón profunda de ser de la empresa (Fernández, 2007) es la maximización a largo plazo del bienestar de todos los stakeholders, cumpliendo con la condición de permitir la consecución de un resultado económico-financiero capaz de llenar suficientemente las expectativas de los accionistas.

Partiendo de los considerandos a los que hemos hecho referencia y dando un paso más en nuestra aproximación al fenómeno, nos topamos con la inesquivable y compleja agenda de enfrentarse con el reto de la sostenibilidad. Porque, como corolario de los párrafos precedentes, podríamos afirmar que la sociedad parece estar demandando de las empresas unos objetivos, por una parte, muy amplios y, por otra, unas pautas de comportamiento muy concretas para tratar de alcanzarlos. Naturalmente, los ejecutivos empresariales debieran prestar atención a estos requerimientos si de veras aspiran que sus empresas se mantengan y, eventualmente se expandan: Precisan, a este respecto una suerte de legitimación social o, como se dice en el ámbito anglosajón, alguna especie de licence to operate. Y por lo que vamos viendo en los últimos tiempos, estas exigencias incluyen no sólo cuestiones económicas sino también otras consideraciones de carácter social y medioambiental.

Pues bien, atender a esta triple cuenta de resultados cuando se genera progreso en el largo plazo es a lo que podríamos denominar Desarrollo Sostenible, es decir, el proceso a largo plazo generador de progreso económico, social, cultural, político y medioambiental para las comunidades humanas (desarrollo polivalente de carácter integral). Aplicado a la empresa se trata de un desarrollo o visión que tiene en cuenta una famosa triple dimensión (Elkington, 1999), económica, social y medioambiental (triple bottom line o 3P's Model, en inglés).

Al demandar que una empresa sea sostenible, se le está pidiendo que satisfaga las necesidades del presente sin comprometer las necesidades de generaciones futuras. Esto, por lo demás, implica que los recursos naturales sean utilizados de tal manera que sea posible su reposición. Pudiera extrañar, en este sentido, que las preocupaciones actuales incluyan no sólo la utilización de recursos agotables (renovables y no renovables) sino también la de recursos inagotables, como el agua. Ello es debido a que el modo en que se viene haciendo uso de este recurso está provocando una degradación en su calidad que tiene un impacto directo sobre el medio ambiente. Por ello, mantener no sólo la cantidad sino la calidad de los recursos es esencial para garantizar la diversidad biológica del planeta.

Ahora bien, para responder a estas nuevas inquietudes las empresas y muchos organismos e iniciativas públicas y privadas están dedicando esfuerzos a re- 
plantear estrategias que permitan la reducción del impacto medioambiental y, al mismo tiempo, promuevan la utilización eficaz de los recursos naturales. $\mathrm{O}$ dicho de otra manera: Que se garantice la disponibilidad de los recursos; que se gestione el impacto ambiental derivado de su utilización. A modo de mnemotecnia suele aludirse a las cuatro erres del enfoque de la sostenibilidad; es a saber: Replantear, Reducir, Reutilizar y Reciclar.

\section{2. ¿Qué es y qué no es la RSE? Deslindes, definiciones y estrategias}

Consideramos que la RSE es y forma parte intrínseca de la empresa y la gestión; que ha de estar ínsita en la propia entraña y dinámica del negocio; que ha de constituir el cañamazo y la urdimbre sobre la que se monten las políticas, las estrategias y las estructuras. A sensu contrario, entendemos que todo lo que sea conceptualizar la RSE como algo añadido, ajeno a la propia razón de ser y al modo de operar de la empresa supone una distorsión del enfoque y una mala interpretación de lo que aquélla significa.

Por desgracia suele ser bastante común caer en este error de enfoque. En consecuencia, estimamos conveniente empezar saliendo al paso y delimitando en forma negativa qué $N O$ es la RSE o, en todo caso, qué no agota del todo lo que la RSE representa. La RSE no es (sólo, ni principalmente) filantropía empresarial; tampoco equivale, sin más a acción social de la empresa (donaciones, patrocinios, mecenazgos, colaboraciones con instituciones y ONG). No es el intento - reactivo - de reparar daños causados de manera más o menos querida, y de la que se siente o puede ser motejada de «responsable». No es — desde luego - una carga más, un nuevo obstáculo que saltar para entrar en la esfera de lo políticamente correcto. Pero, desde el otro punto de vista, tampoco es puro marketing ni debiera ser, sin más, una mera estrategia publicitaria. Por supuesto, no es nada que se pueda anclar cómodamente en el corto plazo, ni se trata una moda, sin más; ni algo que pueda opcionalmente ser o no ser asumido por las empresas. Si tampoco es un departamento más - en la grandes corporaciones-, la pregunta se impone por sí sola:

¿Qué es, entonces, la RSE?

Lo diremos de una manera propositiva: La RSE es la oportunidad más grande para llegar a ser cada día más y cada vez mejor empresa. Constituye un reto de innovación y una gran ocasión para configurar un proyecto organizativo ilusionante, capaz de atraer y retener a los mejores, desde el respeto a la persona, la implicación en lo social y la búsqueda sostenible de la rentabilidad y el desarrollo económico, en aras del bien común.

A efectos de precisar lo que hemos querido dar a entender en el párrafo anterior, transcribimos una definición de gran consenso: «La RSE es, además del cumplimiento estricto de las obligaciones legales vigentes, la integración voluntaria en su gobierno y gestión, en su estrategia, políticas y procedimientos, 
de las preocupaciones sociales, laborales, medioambientales y de respeto a los derechos humanos que surgen de la relación y el diálogo transparentes con sus grupos de interés, responsabilizándose así de las consecuencias y los impactos que se deriven de sus acciones».

Esta definición con la que el Foro de Expertos de la $\mathrm{RSC}^{1}$, promovido por el Ministerio de Trabajo y Asuntos Sociales definía un fenómeno que estaba ya tomando forma en las empresas, parte de otra realizada previamente por la Unión Europea (Unión Europea, 2001) y recogida en un conocido Libro Verde sobre RSE. Analizándola en detalle encontramos algunas características que merece la pena subrayar: (1) La RSE va más allá de lo que exigen «las obligaciones legales». Es decir, el cumplimiento de la ley es condición necesaria para ser considerada una empresa responsable, pero no suficiente. (2) La RSE es de «integración voluntaria»; (3) ha de «integrarse en su gobierno y en su gestión, a través de su estrategia, sus políticas y sus proyectos». O, lo que es lo mismo, debe tener una aplicación transversal en toda la organización. Y (4) dicha integración ha de «incluir preocupaciones sociales, laborales, medioambientales y de respeto a los derechos humanos», lo que implica un enfoque tridimensional que se conoce como la triple bottom line.

Ahora bien, contar con esta definición, que parece actualmente aceptada por todos, no significa que la RSE haya sido ya entendida como una forma de gestionar las empresas. Ciertamente, aún hay un alto porcentaje de la población empresaria que no acaba de tener muy clara la precisión del concepto.

En todo caso, la RSE supone una manera de gestionar las organizaciones que modifica y supera el concepto neoclásico de empresa según el cual su único objetivo era «maximizar el beneficio para el accionista». Que la empresa es una «unidad de organización dedicada a actividades industriales, mercantiles o de prestación de servicios con fines lucrativos» ${ }^{2}$ es una definición por todos aceptada. Que las empresas se crean para organizar una serie de recursos (financieros, humanos, materiales, naturales,...) de tal manera que permitan obtener un beneficio económico en contrapartida, queda implícito en la afirmación anterior. Resulta, pues, evidente que una empresa que no arroje unos resultados económicos positivos no es sostenible en el largo plazo. Pero, ¿es ése el único tipo de beneficio que la sociedad espera de una empresa?

Cada empresa habría de descubrir su razón profunda de ser, su contribución específica a la sociedad; es lo que se conoce en estrategia empresarial como la misión de la empresa. Su formulación debe responder a preguntas tales como ¿qué hacemos en esta empresa? ¿para qué y para quién lo hacemos? ¿qué aportamos a la sociedad? Cuanto más «diferencial» resulte el valor de esta apor-

1 Este foro de expertos finalizó su trabajo en junio de 2006 y en febrero de 2008 fue constituido el Consejo Estatal de la RSE, un órgano adscrito al Ministerio de Trabajo e Inmigración que tendrá un carácter "asesor y consultivo", ya que se encarga de apoyar e impulsar las políticas públicas de apoyo y promoción de la responsabilidad social.

2 Diccionario de la Real Academia de la Lengua Española. 
tación a la sociedad, la empresa contará con un mayor grado de «autorización» para sobrevivir.

Además de la misión, es preciso definir cuál es la visión de la empresa, es decir, dónde pretende estar en un futuro. Para poder llegar a ese punto, deberá actuar de una determinada manera, de acuerdo a los valores con los que se identifica y que deberán marcar las conductas de todos los que trabajan en la empresa y la representan. El conjunto de esos valores y el cómo se aplican en la rutina diaria de la organización configurarán su cultura empresarial. Muchas empresas establecen mecanismos de control para asegurarse que el comportamiento de todos en la organización está alineado con sus planteamientos. Entre ellos, los Códigos Éticos o Códigos de Conducta han ganado protagonismo como pauta de actuación para los empleados y directivos.

Asentados así los pilares de la empresa, habrá que ir marcando los pasos que van a permitir materializar la misión (objetivos, políticas y planes de acción), definidas a través de su estrategia corporativa. De todas las decisiones que se vayan tomando para hacer realidad lo planificado — desde el nivel más estratégico al más operativo- la empresa tendrá que rendir cuentas ante la sociedad.

Pero, ¿cuáles son aquellas cuestiones sobre las que la sociedad va a exigir responsabilidades a la empresa? ¿En qué aspectos la va a someter a un mayor escrutinio? Esto puede depender de muchas variables en función del contexto en el que la empresa opere, cambiante según el momento histórico, y que actualmente cuenta con unas peculiaridades que analizaremos en el siguiente apartado. Sean cuales sean, si la empresa no satisface las expectativas del mercado en estos puntos y en el contexto que le toca vivir, está condenada a desaparecer.

\section{Argumentos para una gestión responsable}

No son pocos los académicos y ejecutivos contrarios al fomento de la RSE dentro de las organizaciones. El principal paladín de esta corriente fue el Premio Nobel Milton Friedman (1970), afamado economista autor de un artículo cuyo título encierra la clave de su enfoque de RSE: «La RSC de la empresa consiste en incrementar sus beneficios».

Según Friedman y sus seguidores carece de sentido que la empresa dedique recursos a la gestión de la RSE y argumentan:

Los directivos trabajan para defender los intereses de los accionistas, de acuerdo con la Teoría de Agencia que rige el gobierno de buena parte de las empresas, especialmente de las cotizadas.

Estarían invadiendo competencias que ni les corresponden ni para las que están preparados. Si bien aquí, probablemente se está produciendo una confusión entre la RSE y la Acción Social, que aclararemos más adelante. 
Gastan dinero de terceros (accionistas, empleados y clientes. Este enfoque se fundamenta en interpretar la RSE como un gasto y no como una inversión en el largo plazo, capaz de repercutir positivamente en los resultados de la compañía.

Quienes invierten en RSE están en desventaja frente a empresas competidoras free-riders en el corto plazo. Probablemente sea éste el argumento más fuerte de los presentados aquí, pues es totalmente cierta esta afirmación y no cabe objetar nada: quienes dedican recursos - lo que siempre lleva aparejado un coste económico- a gestionarse siguiendo pautas de RSE están realizando una aportación al Desarrollo Sostenible de toda la sociedad del que se van a ver beneficiados incluso aquellos competidores que hayan renunciado a gestionar de esta forma. Pero el cuestionamiento ético de este aprovechamiento ilícito es evidente.

Sin embargo, siempre hizo referencia Friedman a la necesidad de respetar la ética y las reglas del juego, quitando así legitimidad a quienes defienden que el beneficio económico prima por encima de todo.

Por el contrario, las razones que exponen quienes impregnan su filosofía de gestión empresarial con la RSE pueden clasificarse de distinta manera en función de la motivación en la que basen su decisión. De este modo, encontramos cuatro tipos de argumentos:

a) Motivación Moral (Moral Case)

a. Imperativo categórico kantiano: «actúa siempre de manera que la máxima de tu actuación se convierta en ley universal»; «trata a los demás como un fin en sí mismo».

b. Existen contratos incompletos en la economía que deben ser equilibrados en pro de un mundo más justo y solidario.

b) Motivación Social (Social Case)

a. La empresa debe realizar su aportación al Bien Común.

b. La actividad empresarial provoca externalidades negativas que debe compensar para alcanzar el equilibrio sostenible del planeta.

c) Motivación Económica (Economic Case)

a. Reducir riesgos no diversificables (ecoeficiencia).

b. Potenciar el aprendizaje organizacional (innovación).

d) Motivación de Negocio (Business Case)

a. Reducir los riesgos reputacionales minimizando los conflictos con los grupos de interés.

b. Acumular intangibles para incidir positivamente en la reputación y en la imagen comercial de la compañía. 
c. Obtener, en el largo plazo, rentabilidad financiera de la inversión (sin contrastar).

Independientemente de los motivos que empujan a las empresas a incorporar la RSE a su gestión, los beneficios recíprocos que se pueden llegar a obtener son muy diversos y tienen un efecto multiplicador en el valor de la compañía en el largo plazo. Fidelidad de los clientes, diferenciación de la competencia, atracción de talento, captación de inversiones o de consumos «responsables», mejora en el clima laboral,... son algunas de las ventajas parciales que, en definitiva, conducen a la obtención de una mayor legitimidad por parte de la sociedad en general. Y, como se echa de ver, esta manera de abordar la gestión está más en consonancia con las expectativas que la ciudadanía va teniendo, a medida que va tomando más conciencia de los rasgos del panorama que nos acompaña en estos inicios del nuevo siglo y a los que hicimos referencia al principio de este trabajo.

\section{La dimensión estratégica de la RSE y las herramientas de gestión}

Antes de ponerse manos a la obra en la implantación de la RSE es preciso que la empresa evalúe sus características intrínsecas y los recursos de que dispone para alcanzar un determinado grado de compromiso en su desarrollo. No resulta efectivo copiar lo que otras empresas están desarrollando en responsabilidad social, pues cada organización tiene sus propias peculiaridades y circunstancias. Es por ello absolutamente imprescindible realizar una profunda introspección antes de tomar ninguna decisión estratégica y, posteriormente, adaptar la implantación a la idiosincrasia organizativa.

Con todo, hay algunos modelos de gestión estandarizados que pueden servir de guía a las empresas en su camino hacia la implantación de la RSE. Los más conocidos son, en España, el Sistema de Gestión Ética SGE21 que promueve Forética y, fuera de nuestras fronteras, los sistemas AA1000 y SA8000. Todos ellos suponen una referencia para aquellas empresas que quieran incorporar sistemas estandarizados de gestión que incorporen criterios éticos y responsables. Cualquiera de ellos es certificable a través de agencias autorizadas que se encargan de verificar que los sistemas de las empresas se corresponden con las indicaciones de estos estándares, consultables a través de las referencias bibliográficas que incorporamos al final de este trabajo.

En cualquier caso, sea la implantación de la RSE en la estrategia organizativa resultado del seguimiento de un modelo estandarizado o una propuesta propia de la empresa, las características que debe el proceso de implantación debiera contemplar, serían las siguientes:

- Debe tratarse de una implantación transversal dentro de la organización que abarque todas las áreas de la empresa a todos los niveles.

- Ha de contar con el apoyo explícito de la Alta Dirección. 
- Tendrá que promover la participación activa y convencida de todos.

- Su desarrollo deberá hacerse a través de procesos sistemáticos, transparentes y fiables, siguiendo un calendario realista.

- La implicación de los empleados resulta crítica, por lo que habrá que prestarles especial atención para evitar incoherencias, recelos y reticencias.

- Y, por supuesto, como en cualquier implantación de procesos organizativos, es necesario partir de un meditado plan de trabajo que deje a la improvisación el mínimo indispensable.

Es preciso empezar con un estudio del entorno en el que la empresa opera, la competencia y las fortalezas y debilidades de la organización, que pudiera realizarse mediante un análisis DAFO. La empresa, por lo demás, debiera tener formulada expresamente y, en la medida de lo posible, asumida cuál es su aportación a la sociedad. Es decir, tal como señalábamos anteriormente, deberá conocer su misión, su visión y sus valores.

Ya se ha indicado más arriba la importancia de establecer un diálogo sincero y fluido con los stakeholders para identificar sus expectativas y encontrar «espacios de beneficio mutuo». Ahora bien, llegado el momento de hacer operativos los planteamientos estratégicos tiene la organización que marcarse unas metas concretas y unos planes de actuación para alcanzarlos. Todo ello, por lo demás, tiene que ser concretado de manera mensurable, con metas cuantificables, indicadores de desempeño y plazos.

Obviamente, si la planificación estratégica no es nunca un proceso fácil, menos aún va a resultar en este ámbito, toda vez que se trata de medir un desempeño social que es intangible. Ahora bien, sólo desde ese esfuerzo será posible realizar una gestión y un seguimiento adecuados y con datos fiables tanto para la propia organización como para toda la sociedad. En este sentido, la aparición de algunos índices bursátiles, como el Dow Jones Sustainability Index o el Footsie4Good han supuesto un inestimable esfuerzo para contribuir a la medición de la RSE de las empresas. Sus analistas financieros valoran desde la TBL la gestión responsable de las empresas que incluyen en sus índices, lo que permite a su vez a otros analistas de fondos de inversión y, en general, a otros inversores tomar decisiones.

Como ya dijimos, hay muchas formas de enfocar las políticas de RSE. Algunas están más orientadas hacia el exterior — tales como, por ejemplo, las políticas de Acción Social o de Relaciones Públicas - y otras a la parte interna de la empresa, tales como podrían ser las políticas de Recursos Humanos o de Organización de la Producción.

Las políticas, como es sabido, se definen por áreas pero para hacerlas realidad hay que concretarlas en proyectos ejecutables y programas concretos que permitan conseguir las metas que de manera cuantitativa se hayan marcado dentro 
de cada política. Y como no podría ser de otra manera, de que los resultados alcanzados a través de esas políticas y concretados a través de los pertinentes proyectos y programas sean fiables, va a depender en buena medida el grado de confianza que la sociedad deposite en la empresa.

Por ello, un factor que puede contribuir a aumentar la certidumbre sobre la gestión empresarial son las verificaciones que entidades externas puedan realizar para garantizar la fiabilidad de los datos. Estas auditorías externas garantizan a los grupos de interés una información veraz y útil para cada uno de ellos en su relación con la empresa. Para la realización de estas auditorías, algunas instituciones han ido desarrollando modelos de gestión verificables y que las empresas que lo consideran conveniente están demandando como aseguramiento externo, objetivo, imparcial y sistemático de una gestión ética, socialmente responsable y sostenible.

Aunque no procede entrar por menudo en el detalle de los mismos - nos remitimos a las referencias bibliográficas al final del trabajo para que el lector interesado profundice en ellos- mencionaremos como ejemplos significativos el modelo AA1000, el SA8000, las normas SGE21 (española, creada por Forética) y las recomendaciones y guías de la IS0 26000, recientemente aprobada. Aunque todos ellos son, por supuesto, de carácter estrictamente voluntario, facilitan a las empresas, un modelo estandarizado para implantar la RSE; y suministran a los diferentes grupos de interés, una herramienta homogénea y, por ello, siempre útil a la hora de interpretar y comparar la calidad de la gestión y del desempeño de las empresas.

Esta labor de comunicación es sumamente crucial. De hecho, sin ella no acabaría cerrado a cabalidad el círculo estratégico de una gestión responsable, pues, al margen de dicha información, muy difícilmente podrían los stakeholders comprobar si sus expectativas han quedado satisfechas o siquiera acometidas por parte de la organización. En este sentido, la elaboración de las Memorias de RSE o de los Informes de Sostenibilidad resulta una herramienta muy apropiada para divulgar todo el trabajo que la empresa lleva a efecto en relación a la sostenibilidad, toda vez que, en cualquiera de sus versiones se tiende a recoger información sobre la triple cuenta de resultados económica, social y medioambiental a la que ya hemos hecho referencia en repetidas ocasiones a lo largo de este artículo.

Como aún es poco frecuente la redacción de este tipo de documentos y como es previsible una generalización de los mismos en el inmediato futuro, no es despreciable la opción de considerar, a este respecto, un nicho de empleo para egresados de las facultades universitarias de las ciencias de la comunicación. 


\section{La institucionalización ética de la Responsabilidad Social}

El escenario en el que nos movemos y que comenzamos describiendo al inicio de estas reflexiones; las expectativas que la sociedad tiene respecto a las empresas y organizaciones económicas; las estrategias que las empresas más avanzadas están poniendo en marcha respecto a la Sostenibilidad y la Ética Empresarial, van dando como resultante una suerte de institucionalización de la RSE. Algunas de las más conocidas iniciativas son las siguientes:

\subsection{Los Principios para los Negocios de la Caux Round Table}

La Caux Round Table fue fundada en 1986 con el propósito de reducir las tensiones en el comercio internacional, desarrollar unas relaciones económicas y sociales constructivas entre los países miembros y asumir el papel que a las empresas les corresponde desempeñar en pro de la paz y la estabilidad internacionales.

Los grandes ejes axiológicos de Caux son dos valores, oriundos de dos tradiciones culturales distintas: el concepto de kyosei — término japonés que significa algo así como «vivir y trabajar juntos para el bien común»-y la apelación a la dignidad de la persona humana, de cariz cristiano occidental. Sobre ellos se articulan los Principios correspondientes: 1. La responsabilidad de las empresas: más allá de los accionistas, hacia todas las personas involucradas en los negocios. 2. El impacto social y económico de las empresas: hacia la innovación, la justicia y una comunidad mundial. 3. La conducta de las empresas: más allá de la letra de la ley, hacia un espíritu de confianza. 4. Respeto a la legalidad. 5. Apoyo al comercio multilateral. 6. Respeto al medio ambiente. 7. Prevención de operaciones ilícitas.

Estos principios se proponen como guías para que las empresas desarrollen, contrasten, pongan en funcionamiento y evalúen sus propios principios y traten de llevarlos a la práctica del día a día.

\subsection{Directrices de la OCDE para las Empresas Multinacionales}

Ya en 1976, la Organización para la Cooperación y el Desarrollo Económico - OCDE - había elaborado una primera versión de las directrices para las empresas multinacionales. Sin embargo, a tono con el calado que iban conociendo los procesos globalizadores de la economía, sus miembros hubieron de llevar a cabo en el año 2000 una revisión profunda, para ajustarlas a las circunstancias actuales, sentar bases más firmes y proponer valores seguros sobre los que articular las transacciones. Aunque no tienen el carácter coercitivo de la ley -ni, por supuesto, son sustitutivas de ordenamiento jurídico alguno-, las directrices constituyen un mensaje importante como recomendaciones que los treinta y tres gobiernos que conforman la OCDE envían a las empresas multinacionales. 
Las directrices pueden ser resumidas en los siguientes términos: 1. Se trata de principios voluntarios y estándares de conducta empresarial responsable, compatibles en todo caso con los imperativos legales. 2. Las empresas deben someterse a la legalidad de los países en los que operan y tomar en consideración los intereses de los múltiples stakeholders. 3. Las empresas deben suministrar adecuada, regular y fielmente la información relevante respecto a sus operaciones, estructura, funcionamiento y situación financiera. 4. Las empresas deberán respetar los derechos de los trabajadores. 5. Deberán tomar en consideración la necesidad de proteger el medio ambiente, la salud pública y la seguridad, buscando un desarrollo sostenible. 6. Desterrarán de sus prácticas el soborno, en cualquiera de sus formas. 7. Velarán por los intereses de los consumidores. 8. Tratarán de llevar a cabo transferencia de tecnología y apostarán por la formación, la investigación y el desarrollo. 9. La competencia será leal y se evitarán prácticas restrictivas a la misma. 10. Contribuirán lealmente mediante el pago de los impuestos a que, en su caso, haya lugar.

\subsection{La Global Reporting Initiative}

La Global Reporting Initiative se define a sí misma como «un proceso institucional multi-stakeholder» cuya misión consiste en desarrollar y distribuir directrices universalmente aplicables para llevar a efecto la información respecto a la sostenibilidad. Dichas directrices tienen también carácter voluntario.

Por ello, las empresas que quieran, pueden adoptarlas a la hora de suministrar a la opinión pública información sobre los aspectos económicos, sociales y medioambientales de sus actividades, productos y servicios. La Global Reporting Initiative incorpora la participación activa de representantes del mundo de la empresa y las finanzas, así como de representantes de organizaciones ecologistas, de lucha por los derechos humanos, de trabajadores y de centros de investigación de todo el mundo.

La Global Reporting Initiative comenzó su existencia en 1997, de la mano de la Coalition for Environmentally Responsible Economies (CERES). Desde el pasado año 2002 es un organismo independiente, colaborador oficial del Programme Environment de las Naciones Unidas (UNEP) y uno de los centros que cooperan dentro del marco del Pacto Mundial de las Naciones Unidas (Global Compact), al que nos referiremos inmediatamente.

\subsection{El Pacto Mundial de las Naciones Unidas}

La idea de establecer la red denominada Global Compact fue propuesta por el entonces Secretario General de la ONU, Kofi Annan, en enero de 1999 y tomó definitiva forma en Nueva York el 26 de julio de 2000. 
Se trata de una iniciativa que establece una red entre empresas, gobiernos, sociedad civil y la propia ONU, aglutinados en torno a diez principios, de voluntaria observancia, agrupados en cuatro categorías: derechos humanos, relaciones laborales - libertad de asociación, eliminación de los trabajos forzados y del trabajo infantil, lucha contra la discriminación - ecología — responsabilidad medioambiental - y lucha contra la corrupción.

De lo que se trata, en definitiva, es de concienciar a las empresas para que, integrando los citados nueve principios en sus estrategias y operaciones, actúen como agentes capaces de contribuir a solucionar los problemas y a enfrentarse con los retos que la globalización plantea, en el marco de una economía inclusiva y sostenible.

\subsection{Las propuestas de la Unión Europea y su recepción en España}

La Cumbre de Lisboa puso en la agenda de la Comisión un objetivo estratégico de hondo calado y amplia significación económica y social: «convertir a la Unión Europea en 2010 en la economía del conocimiento más competitiva y dinámica del mundo, capaz de crecer económicamente de manera sostenible, con más y mejores empleos y con mayor cohesión social».

En este contexto ha de ser enmarcada la publicación, a la que más arriba hemos hecho ya referencia, del Libro Verde de la Comisión Europea: Fomentar un marco europeo para la responsabilidad social de la empresa (2001); el subsiguiente debate llevado a efecto en los distintos países miembros y las reacciones por parte de las empresas y otros interlocutores interesados - asociaciones, escuelas de negocios, personas individuales-. Y, sin duda, es desde esta clave desde donde ha de ser leída la Comunicación de la Comisión relativa a la responsabilidad social de las empresas: una contribución empresarial al desarrollo sostenible, de julio de 2002; así como los demás documentos de avance.

Aparte de lo ya señalado, existen en España otras iniciativas - unas procedentes del ámbito académico, lato sensu; otras, con orígenes más bien centrados en las propias empresas - en materia de RSE. Ello es indicio, una vez más, de la preocupación e interés con que se aborda el fenómeno y d, si bien en una forma más evolucionada. Lo que, por usar la jerga al uso, pudiéramos muy adecuadamente tildar de versión dos punto cero de la RSE y que, como ya se recogía en otro lugar (Andreu y Fernández, 2011), pudiera alinearse con la búsqueda de una sostenibilidad ampliamente entendida.

Entre las iniciativas originadas en el ámbito empresarial, merecen ser destacadas las siguientes: la Comisión de Investigación de AECA - Asociación Española de Contabilidad y Administración de Empresas (www.aeca.es) - sobre Responsabilidad Social Corporativa; el Foro de Reputación Corporativa (www.reputacioncorporativa.org), prolongado a partir de 2011 en el denominado Corporate Excellence. Centre for Reputation Leadership (www.corporateexcellence.com); 
el Foro Empresa y Desarrollo Sostenible (www.foroempresasostenible.org); y el Club de Excelencia en Sostenibilidad (www.clubsostenibilidad.org).

Por lo demás, en línea con la búsqueda de la medición, la auditoría, la verificación y, en su caso, la certificación de los procesos de negocio y administrativos socialmente responsables, se constituyó hace unos años Forética (www.foretica. es), organización a la que también hicimos referencia más arriba. Forética es una asociación patrocinada por un grupo de empresas españolas, que aspira a sensibilizar, informar y a formar en estos asuntos a quienes se sientan interesados por ellos. Realiza diagnósticos y evaluaciones; y promueve la implantación de herramientas de gestión éticas y socialmente responsables, tales como códigos éticos y memorias de RSE.

Desde un punto de vista más institucional, en España, merecen ser destacadas, además, otras dos iniciativas de interés: la subcomisión parlamentaria elaboradora de un interesante Libro Blanco; así como el denominado Foro de Expertos del Ministerio de Trabajo y Asuntos Sociales. El Informe sobre la Responsabilidad Social de las Empresas _Libro Blanco- en España es un informe elaborado por la Subcomisión Parlamentaria de Responsabilidad Social del Congreso de los Diputados para potenciar la RSE. El Libro Blanco recoge una serie de Constataciones y Directrices Generales basadas en el análisis de 59 comparecencias de expertos. Contiene, así mismo, 58 Recomendaciones destinadas a las Administraciones Públicas, empresas, consumidores, inversores y otros agentes de interés. Véase en Boletín Oficial de las Cortes Generales. Congreso de los Diputados. VIII Legislatura. Serie D: General. 4 de agosto de 2006. No 424 . El Foro de Expertos sobre RSE fue constituido el 17 de marzo de 2005 por iniciativa del entonces denominado Ministerio de Trabajo y Asuntos Sociales con la participación de representantes de varios Ministerios y de expertos provenientes de grupos empresariales, organizaciones de la sociedad civil y de la universidad. El Foro de Expertos concluyó sus trabajos el 12 de julio de 2007 sesión en la que se acordó el documento Las políticas públicas de fomento y desarrollo de la RSE en España. Este documento se suma a otros tres anteriores, dando como resultado el Informe-conclusiones del Foro de Expertos sobre RSE. Puede consultarse en: http://www.mtin.es/empleo/economia-soc/cuerpo.htm

Los resultados de estos trabajos, naturalmente, han ido generalizando entre nosotros el discurso de la RSE y la pertinencia de avanzar en planteamientos prácticos al respecto. Tal, por ejemplo, la creación del Consejo Estatal de RSE que empezó a operar en 2009 y que, lamentablemente, está pasando con más pena que gloria el rubicón del impulso de este tipo de filosofía empresarial entre nosotros. 


\section{Las luces y las sombras de un proceso inacabado}

Señalábamos más arriba cómo se han ido dando pasos a favor de la institucionalización del discurso y las preocupaciones respecto a la RSE a lo largo de estos últimos años. Decíamos también que, con todo y con eso, el resultado final debía ser entendido como escaso e insuficiente, aunque no sea más que por el ridículo número de compañías que han suscrito el Pacto Mundial de la ONU. Se podrá decir que las grandes lo han hecho y que el efecto arrastre vendrá detrás; se podrá argüir que hay otras muchas empresas que, sin tener escrito nada, se comportan con altas dosis de calidad ética y con gran responsabilidad en sus relaciones mercantiles... Y, sin duda, es cierto. Pero, en todo caso, no deja de ser llamativo el dato: Un porcentaje ridículo de las empresas del mundo, sólo una parte minúscula, ha optado formalmente por una apuesta decidida a favor de la sostenibilidad de los procesos, el enfoque a largo plazo, la preocupación por el respeto a los derechos humanos, laborales y medioambientales... Sólo unas pocas compañías en estos momentos de crisis parecen haber entendido que la RSE es algo netamente esencial en la dinámica del negocio. Más allá de la tesis separatista que ve al negocio ir por un lado y luego - si las vacas son gordas y las cosas se dan bien- se aplicarán algunos recursos, de manera extraordinaria y siempre exterior y tangencial al quehacer del negocio, en acción social... En definitiva: el balance, en nuestra opinión, es pobre. No queremos decir, en modo alguno, que sea negativo. Es, simplemente, escaso, insuficiente, escaso, menguado, pobre.

Además, corre el peligro de convertirse en ideológico: en encubrir una realidad injusta con un discurso ficticio que canta las loas de lo bien que se está y las excelentes prácticas de los agentes más proactivos. Y este aspecto glorioso es aún más dañino que el primero, el de la insuficiencia, porque distrae de la búsqueda de objetivos inaplazables en un mundo que necesita imperiosamente salir de la crisis y sentar las bases para un futuro sostenible, justo y, en la medida de lo posible, armónico. En ello nos va la supervivencia a medio plazo y de ahí emana una inaplazable cuestión moral que apunta hacia nuestra responsabilidad - personal y organizativa- respecto al planeta y a las generaciones futuras.

\section{Conclusión}

Las empresas no viven en el vacío, sino que nacen y se desarrollan en un contexto cada vez más complejo. Por una parte, es la actual circunstancia, tremendamente turbulenta en lo económico, cada día más dinámica en lo sociocultural, crecientemente preocupada por nuevos retos y problemas - entre otros: el cambio climático, los fenómenos migratorios, el proceso de globalización, la brecha digital, el choque de civilizaciones-; y, en paralelo, por otra parte, vivimos en un momento donde la gente - al menos las elites cultivadas del primer mundo que, por lo demás, son quienes tienen mayor poder de voto monetario, tanto de compra cuanto de inversión- parece ser cada vez menos propensa a comulgar 
con las ruedas de ciertos molinos... y pide, empieza a exigir, que quien pueda estar en condiciones de echar una mano para ver de solventar los grandes problemas con los que nos toca lidiar en este comienzo de milenio, lo hagan con decisión.

Las empresas, por su lado, empiezan a tomar nota de esta hodierna realidad. Son conscientes del nuevo escenario: abierto, desigual, poco o escasamente regulado, sin referentes o con muy tenues líneas marcando el campo de juego. O sea: son conscientes de la estupenda ocasión para, pescando en río revuelto, amonedar montañas de euros... ya sea por lo suave - aprovechando resquicios, jugando con ventaja-, ya sea desde opciones más agresivas — corrompiendo, sobornando, pisoteando los derechos humanos más elementales, atentando contra el común patrimonio que el entorno ecológico supone-, desatendiendo, en todo caso, las implacables voces de la conciencia moral... a mayor gloria del becerro de oro.

La otra cara de la moneda, sin embargo, hace que las empresas tomen también conciencia de las nuevas realidades desde otra óptica, con otra perspectiva; y sobre todo, con otra voluntad estratégica. A saber: la de configurarse a ellas mismas como la altura de los tiempos demanda; no sólo en busca de la excelencia económica, sino exquisitamente atentas a la integración —voluntaria- en sus políticas, planes, procedimientos y modus operandi de las preocupaciones sociales y medioambientales.

Pues bien, en nuestra opinión y al hilo de lo dicho hasta aquí, el fenómeno de la RSE ha de ser inscrito en este marco, si de veras queremos ubicarlo en las coordenadas que le corresponden. Constituye, sin duda, todo un modo nuevo de entender la realidad empresarial; configura una manera más compleja - y por ende más real- de enfrentarse con la tarea de la dirección; se alinea con las expectativas, deseos y demandas de muy distintos y polimórficos grupos de interesados. Conforma, en suma, un auténtico capital axiológico que hay que aprender a manejar..., pues, aunque el valor de la Responsabilidad Social no se toca - es sabido que, por definición y por naturaleza, se trata de un intangible-... sí que se siente; sí que se deja notar — tanto cuando abunda, cuanto, sobre todo, cuando brilla por su ausencia. ¿Indicadores al respecto?... No han de faltar, a tenor de toda la panoplia del argumentario en línea con el business case al que arriba aludimos, para encarecer la conveniencia de ser socialmente responsable... A saber: la sostenibilidad de los procesos, la buena reputación, el convertirse en una empresa-imán que atraiga y retenga a los más capaces, la rentabilidad, la permanencia a largo plazo. Sin embargo, siendo ya mucho lo que va supuesto en tal modo de afrontar el fenómeno, no es ni tiene por qué ser ésa la única línea argumental; es también posible, sin duda, dar mayor fuste y altas miras al proceso y no dejar por sistema a la altura de vuelo rasante la argumentación en pro de la RSE, apelando a la sostenibilidad y fundamentándola en la obligación ética de mantener el planeta y no impedir el desarrollo humano de las generaciones futuras. 


\section{Referencias}

\subsection{Referencias bibliográficas}

Andreu Pinillos, Alberto y Fernández Fernández, José Luis (2011). De la RSC a la sostenibilidad corporativa: Una evolución necesaria para la creación de valor. En: Harvard Deusto Business Review, n 207 (diciembre), 4-21.

Elkington, John (1999). Cannibals with Forks: The Triple Bottom Line of $21^{\text {st }}$ Century Business. Capstone: John Wiley \& Sons Ltd.

Fernández Fernández, José Luis (2007). ¿Por qué y cómo gestionar la ética organizativo-empresarial?. En: Bajo Sanjuán, Anna y Villagra García, Nuria (eds.). Evolución conceptual y práctica de una gestión responsable. Memoria Académica Curso 2006-2007. Madrid: Publicaciones de la Universidad Pontificia Comillas.

Freeman, Robert Edward (1984). Strategic Management: A Stakeholder Approach. Boston: Pitman.

Freeman, Robert Edward (1994). The Politics of Stakeholder Theory: Some Future Directions. En: Business Ethics Quarterly, nº 4, 409-421.

Friedman, Milton (1970). The Social Responsibility of Business Is to Increase its Profits. En: The New York Times Magazine, September 13, 1970, 32-33, 122-126.

McGregor, Douglas (1960). The Human Side of the Enterprise. New York: MacGraw-Hill.

Murray, Henry A. (1938). Explorations in Personality. Oxford: Oxford University Press.

Rusconi, Gianfranco (2007). Introduzione. En: Freeman, Robert Edward; Rusconi, Gianfranco y Dorigatti, Michele. Teoria degli Stakeholder. Milano: FrancoAngeli, Fondazione Acli Milanesi, 13-17.

Solomon, Robert. C. (1999). A Better Way to Think About Business. New York: Oxford University Press.

Taylor, Frederick Winslow (1911). Principles of Scientific Management. New York: Harper.

Weber, Max (1968). Economy and Society: An Outline of Interpretative Sociology. New York: Wedminster.

\subsection{Referencias electrónicas citadas en el texto}

AECA - Asociación Española de Contabilidad y Administración de Empresas (www.aeca.es) 
Club de Excelencia en Sostenibilidad (www.clubsostenibilidad.org)

Corporate Excellence. Centre for Reputation Leadership (www.corporateexcellence.com)

Foro Empresa y Desarrollo Sostenible (www.foroempresasostenible.org)

Foro de Reputación Corporativa (www.reputacioncorporativa.org)

\subsection{Referencias de Sistemas de Gestión de la RSE}

AA1000: www.accountability21.net/aa1000series

ISO/WD26000: www.iso.org/sr

SA8000: www.sa8000.org

SGE21: http://www.sge21.foretica.es/es/

\subsection{Referencias electrónicas de interés general}

Consejo Estatal para la RSE: http://www.mtas.es/es/empleo/economia-soc/

RespoSocEmpresas/ConsejoRSE.htm

Declaración de la OIT sobre derechos y principios fundamentales en el trabajo:

http://www.ilo.org/declaration/lang--en/index.htm

Directrices de la OCDE para empresas multinacionales:

http://www.oecd.org/department/0,3355,en_2649_34889_1_1_1_1_1,00.html

Documentos de la Comisión Europea: http://ec.europa.eu/employment_social/ soc-dial/csr/index.htm

Global Compact: www.unglobalcompact.org

Global Reporting Initiative (GRI): www.globalreporting.org

Pacto Mundial: www.pactomundial.org

\section{Referencia de este artículo}

Bajo Sanjuán, Ana; González González, Marta y Fernández Fernández, José Luís (2013). Responsabilidad social y empresa sostenible. En: adComunica. Revista Científica de Estrategias, Tendencias e Innovación en Comunicación, n ${ }^{\circ} 5$. Castellón: Asociación para el Desarrollo de la Comunicación adComunica, Universidad Complutense de Madrid y Universitat Jaume I, 223-243. DOI: http://dx.doi.org/10.6035/21740992.2013.5.14 